\title{
THE NASA SOLAR PROBE MISSION: IN SITU DETERMINATION OF INTERPLANETARY OUT-OF-THE ECLIPTIC AND NEAR-SOLAR DUST ENVIRONMENTS
}

\author{
BRUCE T. TSURUTANI \\ Solar Probe Study Scientist \\ Jet Propulsion Laboratory \\ California Institute of Technology \\ 4800 Oak Grove Drive \\ Pasadena, California 91109
}

\author{
JAMES E. RANDOLPH \\ Solar Probe Study Manager \\ Jet Propulsion Laboratory \\ California Institute of Technology \\ 4800 Oak Grove Drive \\ Pasadena, California 91109
}

and

\begin{abstract}
The NASA Solar Probe mission will be one of the most exciting dust missions ever flown and will lead to a revolutionary advance in our understanding of dust within our solar system. Solar Probe will map the dust environment from the orbit of Jupiter ( 5 AU), to within 4 solar radii of the sun's center. The region between $0.3 \mathrm{AU}$ and $4 R_{S}$ has never been visited before, so the 10 days that the spacecraft spends during each (of the two) orbit is purely exploratory in nature. Solar Probe will also reach heliographic latitudes as high as $\sim 15^{\circ}$ to $28^{\circ}$ above (below) the ecliptic on its trajectory inbound (outbound) to (from) the sun. This, in addition to the ESA/NASA Ulysses mission, will help determine the out-of-the-ecliptic dust environment. A post-perihelion burn will reduce the satellite orbital period to 2.5 years about the sun. A possible extended mission would allow data reception for 2 more revolutions, mapping out a complete solar cycle. Because the near-solar dust environment is not well understood (or is controversial at best), and it is very important to have better knowledge of the dust environment to protect Solar Probe from high velocity dust hits, we urgently request the scientific community to obtain further measurements of the nearsolar dust properties. One prime opportunity is the July 1991 solar eclipse.
\end{abstract}

\section{Introduction}

In the late 1970 's and early 1980 's, NASA studied a mission to go close to the sun, called Starprobe. In this study: 1) fields and charged particles, 2) solar imaging, and 3) drag compensation experiments to study relativistic effects and the solar gravitation quadrupole moment were considered. However, it became apparent that the latter two categories of experiments necessitated complex spacecraft designs and escalated overall costs. Thus, in 1985 a Space Science Board committee of the National Academy of Sciences, recommended that "....a Solar Probe mission whose primary objective is to carry out the first in-situ observations of the solar wind plasma and fields near the source of the wind in the solar atmosphere. Included will be a detailed study of energetic particles which will yield important diagnostic data on particle acceleration processes and coronal structure" [1].

In 1988, NASA selected a Solar Probe science advisory panel, and from 1989 to the present, this group has carried out such studies $[2,3]$. NASA chose experts from many areas of science to cover the anticipated goals. However, a person for dust science was not included, because Solar Probe was initially thought to be primarily a coronal plasma science mission. With help from $\mathrm{M}$. Hanner and H. Zook, a dust instrument is now part of the payload. Similar effort is presently underway towards the possible inclusion of a hard $(>20 \mathrm{keV}) \quad \mathrm{X}$-ray experiment and a 3-dimensional coronal imager.

Solar Probe is presently in NASA's strategic plan for the 1990s. The preliminary design studies are scheduled in the first-half of the 1990s, phase A from 1991-1992 and phase B from 1993-1994. A Project Start is currently scheduled for 1995, completed spacecraft design and fabrication is scheduled for 1999, leading to a launch in September 2000. The first perihelion would occur in June 2006 and a second in December 2008. The satellite will remain in a solar orbit with a 2.5 year period, so an extended mission is possible.

\section{The Solar Probe Mission}

\subsection{EARTH-JUPITER GRAVITY ASSIST ( $\triangle$ V-EJGA) TRAJECTORY}

29

A.C. Levasseur-Regourd and H. Hasegawa (eds.), Origin and Evolution of Interplanetary Dust, 29-32.

(c) 1991 Kluwer Academic Publishers, Printed in Japan. 
Solar Probe will receive two gravitational boosts and three major velocity increments in the proximity of the Earth, Jupiter and the Sun (Figure 1). Following launch, the spacecraft will receive a deep space velocity increment $(\Delta V)$, orbit the sun once and encounter the Earth to have a gravity-assisted flyby and another $\Delta \mathrm{V}$. The spacecraft will then travel to Jupiter (5 AU) experiencing another gravity assist and will drop in towards the sun. At this point Solar Probe will be in a highly elliptical polar orbit with a perihelion of $4 R_{S}$, an aphelion of about $5 \mathrm{AU}$, and a period of about 4 years. After perihelion flyby, a burn will be commanded to decrease the spacecraft velocity and shorten the orbital period. This burn will be commanded when the spacecraft is 10 to $15 \mathrm{R}_{\mathrm{S}}$ past the sun to minimize perihelion science data loss.

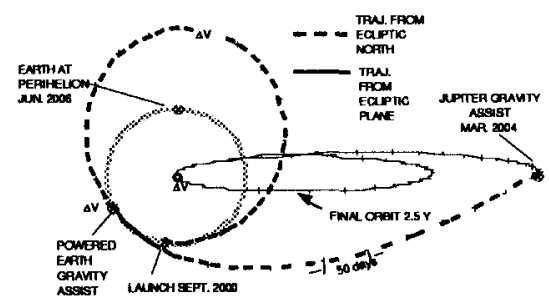

Figure 1. The Solar Probe Trajectory

The near-perihelion passage is shown in Figure 2. Besides monitoring the interplanetary environment out to $5 \mathrm{AU}$, Solar Probe will enter an exploratory phase as it goes inside $0.3 \mathrm{AU}, \mathrm{a}$ region that has never been penetrated before. The spacecraft will spend $\sim 10$ days in this region. Close to the Sun, Solar Probe will first cross the north pole at $\sim 7 R_{s}$, the equator at $4 R_{s}$ and then the south pole at $\sim 7 R_{S}$ all in a span of about 14 hours. The spacecraft orbital plane has been designed to be nearly orthogonal to the Earth-Sun line, so that constant communication with the spacecraft can be maintained. A Ka-band downlink telemetry system will be used to maintain a 70 $\mathrm{kb} / \mathrm{s}$ data stream through the solar coronal (interference) environment near perihelion. The downlink telemetry has higher rate capability (over $200 \mathrm{~kb} / \mathrm{s}$ ) further from the sun. Some of the near perihelion data will be stored on-board and will be telemetered down after perihelion passage.

At $1 \mathrm{AU}$ on the inbound pass, Solar Probe will be at an out-of-the-ecliptic latitude of $15^{\circ}$. This latitude increases with decreasing distance from the sun, reaching $+28^{\circ}$ at $0.3 \mathrm{AU}$ and $+90^{\circ}$ seven hours prior to perihelion. The outbound pass will sample the dust environment at latitudes below the ecliptic plane, symmetric to the inbound pass.

Solar Probe will therefore give dust information from 5 AU to $4 R_{S}$ using a single instrument. Solar Probe, together with the Galileo mission, will solve one of the current problems of instrument intercalibration (see E. Grü [4], for discussion). If the extended mission is approved, then possible solar cycle dust fluence dependences could also be measured.

\subsection{SOLAR PROBE SCIENCE}

The prime Solar Probe mission objective is to determine the mechanisms by which the solar wind plasma is accelerated and heated. For this objective, Solar Probe is targeted to reach or go inside of $4 \mathrm{R}_{S}$, the nominal solar wind sonic point. Inside this theoretical point, the solar wind is subsonic, and outside, it is supersonic. This perihelion distance is also close to the theoretical dust free cavity boundary [5] ( $<4$ to $5 \mathrm{R}_{\mathrm{S}}$ ) so that the dust properties in and near this boundary will also be explored.

Figure 3 gives the current spacecraft configuration. The heat shield is made of carbon-carbon and will reach surface temperatures up to $2100 \mathrm{~K}$ at perihelion. The heat shield will point toward the sun and be designed so that the umbra (shadow) region where the scientific instruments are located, will remain near room temperature $\left(20-30^{\circ} \mathrm{C}\right)$. 


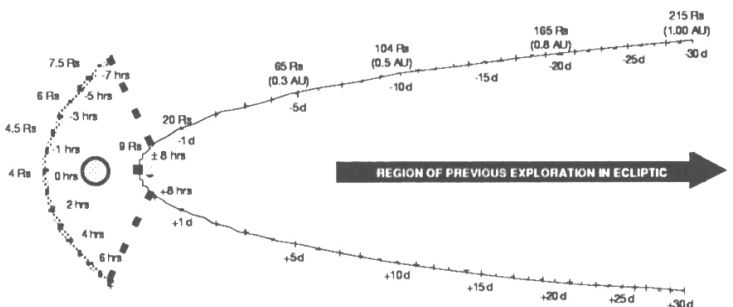

Figure 2. The near perihelion trajectory. The orbit plane is orthogonal to the Earth-Sun line.

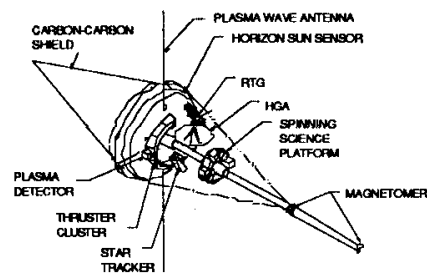

Figure 3. The Solar Probe spacecraft configuration.

Most of the science instruments are located on the science platform, which is moved away from radioisotope thermoelectric generators (RTGs) by a mast. The platform will spin at $60 \mathrm{~mm}$ to allow full three-dimensional distribution measurements once per second. The dust detector is currently located on the platform. It is also possible to place it on the body of the spacecraft, if so desired. The strawman payload is given in [3]. The mass, power, data rate, range, type of detector, field of view, location and heritage for each instrument is given in the reference. At perihelion the spacecraft will be travelling at over $300 \mathrm{~km} / \mathrm{s}$ (and the solar wind only $100-200 \mathrm{~km} / \mathrm{s}$ ), so Solar Probe will be passing through magnetic, plasma and dust structures quite rapidly. It will be necessary to have high time resolution to be able to adequately identify these structures, thus the very high spacecraft data rates.

\section{Dust Hazards}

There are substantial dust hazards, especially in the region near perihelion. At $4 R_{s}$, circularly orbiting dust particles have a velocity of $220 \mathrm{~km} / \mathrm{s}$ while the spacecraft velocity will be over 300 $\mathrm{km} / \mathrm{s}$. It is particularly important to know the direction of the dust orbits. As an example, if they are all in a circumsolar ring in the elliptic plane, then the spacecraft and dust velocity vectors will be orthogonal to each other and the relative velocity will be $380 \mathrm{~km} / \mathrm{s}$. Protection will be needed on only one side of the spacecraft. If, on the other hand, the near solar dust flux is isotropic, then all sides of the spacecraft will have to be protected. The dust impact velocities will range from 90 to $530 \mathrm{~km} \mathrm{~s}^{-1}$.

The near solar dust environment is presently a controversial issue. From balloon-borne and solar eclipse observations of the solar corona [6-10], enhanced near-infrared emissions have been used to argue for the presence of a circumsolar dust ring at $4 \mathrm{R}_{\mathbf{S}}$ (and rings at larger distances). Mizutani et al. [9] has presented a model of a toroidal-shaped cloud $8 \mathrm{Rs}_{\mathrm{s}}$ in diameter and $1 \mathrm{R}_{\mathrm{S}}$ in radius. The particles have a size of $10^{-2} \mathrm{~cm}$ and a number density of $10^{-14} \mathrm{~cm}^{-3}$. Mukai et al. [11] have discussed spatial concentration of dust grains caused by the stabilization of their orbits by sublimation. On the other hand, Mann and Kneissel [12] demonstrate that the near-infrared emission enhancement at $4 R_{S}$ could be explained by optical diffraction instead.

To estimate the dust environment at $4 \mathbf{R}_{S}$ for Solar Probe, we take two different approaches: 1 ) we make an extrapolation from $1 \mathrm{AU}$, and 2) we use the dust ring values of Mizutani et al. [9]. We compare the two sets of numbers below.

To extrapolate from $1 \mathrm{AU}$, we use the interplanetary mass density model of Grün et al. [13]. The radial gradient in densities are assumed to vary as $r^{-1.3}$, where $r$ is the distance from the sun [14]. This dependence was based on Helios zodiacal light observations made for distances in as close as $19 \mathrm{R}_{\mathrm{S}}$ from the sun. (However, we note that recent IRAS results indicate that the particle albedo increases with decreasing solar distance [15-17]. $\mathrm{A} \mathrm{r}^{-1.0}$ scaling law has been derived [17]). The spatial number density at $4 \mathrm{R}_{\mathrm{s}}$ extrapolated from the Grün et al. 1 A.U. values is given in Table $I$. This corresponds to the number densities in the ecliptic plane. 
Table I. The dust number density extrapolated to $4 \mathbf{R}_{\mathrm{S}}$ assuming the Grün et al. [13] $1 \mathrm{AU}$ spectrum and a $\mathrm{r}^{-1.3}$ radial dependence.

$\begin{array}{cc}\text { Mass Range } & \text { Extrapolation to } 4 \mathrm{R}_{\mathbf{S}} \\ 3 \times 10^{-7} \text { to } 3 \times 10^{-6} \mathrm{gm} & \text { S } \\ 3 \times 10^{-6} \text { to } 3 \times 10^{-5} \mathrm{gm} & 1.1 \times 10^{-15} \mathrm{~cm}^{-3} \\ 3 \times 10^{-5} \text { to } 3 \times 10^{-4} \mathrm{gm} & 1.8 \times 10^{-16} \mathrm{~cm}^{-3} \\ & 1.1 \times 10^{-17} \mathrm{~cm}^{-3}\end{array}$

Comparison of the above values to those of Mizutani et al. indicate that the ring model is about 15 times higher in density than the extrapolated values. If a $\mathrm{r}^{-1.0}$ model is used for the extrapolation, then the difference between the ring model and the extrapolation is even greater.

\section{Conclusions}

The Solar Probe mission will be a revolutionary advance in our understanding of dust within the solar system. It will be able to answer the question as to whether there are dust rings near the sun, determine the particulate mass and velocity distributions in the near-solar region and determine the particle spectrum at the boundary(s) to the dust free zone(s). It will also be able to measure the gradient in particles from $4 R_{s}$ to $5 \mathrm{AU}$ and to measure the out-of-the-ecliptic dust distribution. The measurements should provide much of the necessary information to determine the sources of interplanetary dust within the heliosphere.

Acknowledgements. Portions of this research were carried out at the Jet Propulsion Laboratory, California Institute of Technology under contract with the National Aeronautics and Space Administration. We very much appreciate comments and correspondence from $M$. Hanner, B. Kneissel, I. Mann, A. Levasseur-Regourd, P. Lamy and T. Mukai, and the meeting convenors for their kind invitation to speak at the IAU Colloquium. We also appreciate a reading of this manuscript by B. E. Goldstein, (the Solar Probe Deputy Study Scientist) and J. A. M. McDonnell, the referee of the paper, for helpful comments and suggestions.

\section{References}

1. 'An Impl. Plan for Priorities in Sol.-Syst. Space Phys.' (1985), Com. Sol. and Space Phys. of the Space Sci. Bd., Nat. Acad. Press, Wash. D.C.

2. Sol. Probe Miss. Sys. Design Concepts $1989^{\prime}$ (1989), ed. J. E. Randolph, JPL Internal Document D-6798.

3. 'Solar Probe Scientific Report' (1989), JPL Internal Document D-6797.

4. Grün, E., et al. (1991), In-situ space expl. of dust in sol. syst. and init. results from Galileo dust det., in Orig. and Ev. Interpl. Dust, Kluwer, Dordrecht.

5. Lamy, Ph.L.. (1974), 'Interact. of interpl. dust grains with sol. rad. field', Astron. and Astrophy., 35, 197-207.

6. MacQueen, R. M. (1968) 'Infrared obs. of outer sol. cor.', Astrophys. J., 154, 1059-1076.

7. Peterson, A. W. (1969) 'Exp. det. therm. rad. interpl. dust', Astrophys. J., 148, L37-39.

8. Peterson, A. W. (1971), Bull. Amer. Astron. Soc., 3, 500.

9. Mizutani, K. et al. (1984) , Near-infrared obs. circumsol. dust emis. during 1983 sol. eclipse', Nature, 312, 134-136.

10. Mukai, T. (1985), 'On the sol. dust rings', in Props. Interacts. Interpl. Dust, R. H. Giese and P. Lamy, (eds.), D Reidel, 59-62.

11. Mukai, T., (1974), 'On circum. grain mat.', Publ. Astron. Soc. Japan, 26, 445-458.

12. Mann, I. and Kneissel, B. (1991' 'Interpl. dust close to the sun', in Orig. Ev. Interpl. Dust, A. C. Levasseur-Regourd (ed.), Kluwer, Dordrecht.

13. Grün, E., et al. (1985), 'Coll. bal. of meteoric compl.', Icarus, 62, 244-272.

14. Leinert, C., Richter, I., Pitz, E., and Planck, B. (1981) 'The zod. light from 1.0 to $0.3 \mathrm{AU}$ as obs. by Helios space probes', Astron. Astrophys., 103, $177-188$.

15. Good, J. C., et al. (1986), 'IRAS obs. of zod. backgrd.' Adv. Space Res., 6, 83-86.

16. Kneissel, B. and Giese, R. H. (1986), "The impact of IRAS results on 3-D models glob. distr. interpl.. dust' Adv. Space Res., 6, 79-82.

17. Levasseur-Regourd, A. C. and Dumont, R. (1990) 'IRAS obs. zod. backgrd.' Adv. Space Res., 6, 83-86.

18. Levasseur-Regourd, A. C., et al. (1990), 'Dust op. prop.: comp. betw. comet. interpl. grains', Adv. Space Res.' 